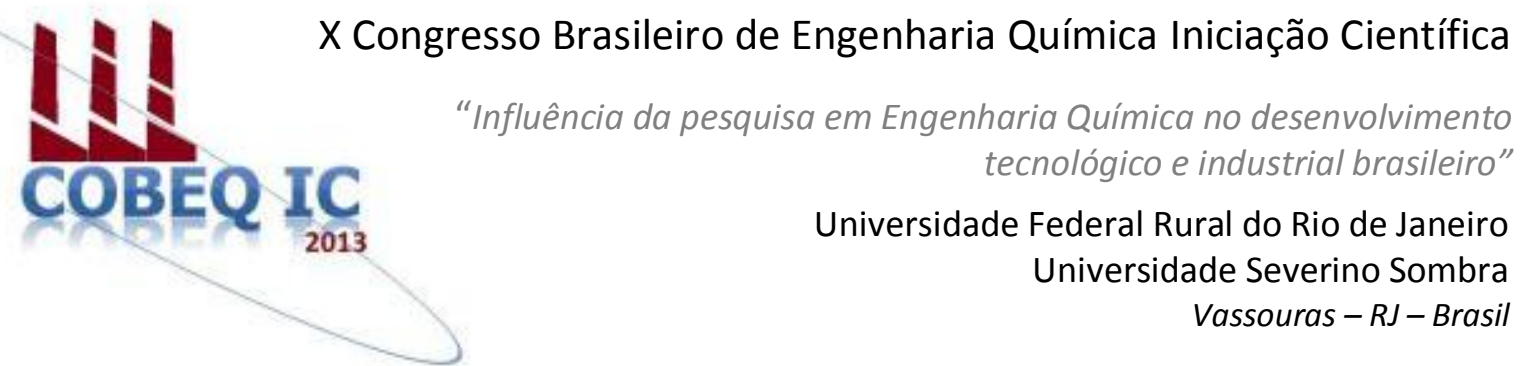

\title{
DISCRIMINAÇÃO DE EQUAÇÕES PARA ISOTERMAS DA UMIDADE DE EQUILÍBRIO DA CASCA DE MACADÂMIA
}

\author{
BARCELOS $^{1}$, K.M.; XAVIER ${ }^{2}$, T.P.; BACELOS ${ }^{2}$, M.S.; BARROZO $^{3}$, M.A.S.; LIRA $^{2 *}$, T.S. \\ ${ }^{1}$ Aluna da UFES $\quad{ }^{2}$ Professor do DETEC/UFES $\quad{ }^{3}$ Professor do FEQ/UFU \\ Departamento de Engenharias e Tecnologia - Universidade Federal do Espírito Santo \\ Endereço-UFES, BR 101 Norte, Km 60, Bairro Litorâneo, São Mateus, CEP. 29.932-540, ES, \\ email: taisa.lira@ufes.br
}

\begin{abstract}
RESUMO - A macadâmia é uma amêndoa com alto valor agregado no mercado mundial e em pleno desenvolvimento no Espírito Santo. Sua utilização pela indústria é ampla, porém, os resíduos de seu beneficiamento, carpelo e a casca, não apresentam grande utilidade no mercado. Esses, entretanto, se processados adequadamente, podem se tornar uma boa alternativa para geração de energia renovável a partir do processo de pirólise. Porém, estudos preliminares, tais como a investigação do comportamento das isotermas de equilíbrio de adsorção, devem ser realizados. Neste trabalho foram realizados experimentos baseados no método estático. Os dados de umidade de equilíbrio foram ajustados às equações de Henderson, Henderson-Thompson, ChungPfost, Chen-Clayton, Halsey modificada, Oswin modificada, Sabbah, Copace e SigmaCopace por meio de regressões não lineares. Para determinar a equação que melhor representa o comportamento experimental foram utilizadas as medidas de curvatura de Bates e Watts e o vício de Box. Na faixa de condições experimentais estudadas, os modelos de Halsey modificada, Copace e Sigma-Copace foram os que melhor se ajustaram ao comportamento da umidade de equilíbrio do material.
\end{abstract}

Palavras chave: determinação de parâmetros, medidas de não linearidade, método estático.

\section{INTRODUÇÃO}

A nogueira macadâmia foi introduzida comercialmente no Brasil no início da década de 90, e atualmente o País é o sétimo maior produtor mundial, com destaque para os estados de São Paulo, Espírito Santo e Bahia. No Espírito Santo, o plantio foi iniciado no final da década de 90 e atualmente, o estado é o segundo maior produtor do Brasil, sendo o município de São Mateus o maior produtor do estado, com aproximadamente 500 hectares de área plantada e produção de 800 toneladas por ano (GLOBO RURAL, 2011).

A macadâmia é um folículo composto por três partes: o carpelo de consistência carnosa e cor verde, a casca que apresenta coloração marrom e encontra-se envolvida pelo carpelo, e no interior, a amêndoa de coloração creme.

A utilização da amêndoa pela indústria é ampla (PIMENTEL, 2007), porém, a 
disposição dos resíduos de cascas ou carpelo tem se tornado um sério problema para as indústrias de processamento da noz, devido ao aumento mundial de sua produção (POINERN et al., 2011). Estes resíduos podem se tornar uma boa alternativa para geração de energia renovável, uma vez que geram produtos com alto poder calorífico a partir da pirólise. No entanto, estudos preliminares, tais como a investigação das isotermas de adsorção, devem ser realizados.

A umidade de equilíbrio de um material é definida como o teor de umidade correspondente ao equilíbrio entre as pressões de vapor da água no material e no meio ambiente. Esta variável é a que determina o teor mínimo de umidade que o material pode atingir em um determinado conjunto de condições operacionais.

As duas técnicas clássicas de determinação de umidade de equilíbrio são através dos métodos estático e dinâmico. $\mathrm{Na}$ primeira, o ar sem movimento circunda $\mathrm{o}$ material e na segunda o fluido é movido mecanicamente. A vantagem do método estático é a maior facilidade de se atingir condições termodinamicamente constantes.

Muitas equações teóricas, semiempíricas e empíricas foram propostas para a estimativa da umidade de equilíbrio. As equações teóricas são baseadas nas conhecidas teorias cinéticas da adsorção, porém não conseguem prever com precisão os valores de umidade de equilíbrio para uma ampla faixa de temperatura e umidade relativa do ar (BARROZO, 1995). Esse fato motivou o aparecimento de vários modelos empíricos e semi-empíricos (Henderson, 1952; HendersonThompson, 1968; Chung Pfost, 1967; Chen Clayton, 1971; Halsey Modificada, 1989; Oswin Modificada, 1989; Sabbah, 1976; Copace, 1995; Sigma Copace, 1995).

Este trabalho foi realizado com o objetivo de estudar as isotermas da umidade de equilíbrio da casca de macadâmia e verificar, dentre as equações encontradas na literatura, aquela que melhor se ajusta ao comportamento experimental.

\section{METODOLOGIA}

As amostras de cascas de macadâmia utilizadas foram cedidas pela Cooperativa Agroindustrial dos Produtores de $\mathrm{Noz}$ Macadâmia (Coopmac), situada no município de São Mateus, ES. O teor de umidade inicial da casca é de $11,7 \pm 0,1 \%$ (b.s.).

$\mathrm{O}$ método adotado para a determinação do teor de umidade no equilíbrio foi o estático, com a utilização de soluções salinas saturadas de Cloreto de Lítio, Acetato de Potássio, Cloreto de Magnésio, Carbonato de Potássio, Nitrato de Sódio, Cloreto de Sódio e Cloreto de Potássio. Os experimentos (em triplicata) foram conduzidos nas temperaturas de 20, 30, 40,50 e $60{ }^{\circ} \mathrm{C}$. As umidades relativas de cada solução saturada nas diferentes temperaturas são mostradas na Tabela 1 .

Tabela 1 - Variação da umidade relativa com a temperatura.

\begin{tabular}{cccccc}
\hline Sal & $\mathbf{2 0}^{\circ} \mathbf{C}$ & $\mathbf{3 0}^{\circ} \mathbf{C}$ & $\mathbf{4 0}^{\circ} \mathbf{C}$ & $\mathbf{5 0}^{\circ} \mathbf{C}$ & $\mathbf{6 0}^{\circ} \mathbf{C}$ \\
\hline $\mathbf{L i C l}$ & 0,117 & 0,111 & 0,105 & 0,100 & 0,096 \\
$\mathbf{C H}_{3} \mathbf{C O}_{2} \mathbf{K}$ & 0,249 & 0,226 & 0,206 & 0,189 & 0,175 \\
$\mathbf{M g C l}_{\mathbf{2}}$ & 0,334 & 0,323 & 0,313 & 0,304 & 0,295 \\
$\mathbf{K}_{\mathbf{2}} \mathbf{C O}_{3}$ & 0,447 & 0,440 & 0,433 & 0,427 & 0,421 \\
$\mathbf{N a N O}_{2}$ & 0,675 & 0,643 & 0,614 & 0,588 & 0,564 \\
$\mathbf{N a C l}$ & 0,772 & 0,752 & 0,734 & 0,718 & 0,703 \\
$\mathbf{K C l}$ & 0,873 & 0,837 & 0,805 & 0,777 & 0,751
\end{tabular}

Inicialmente, a amostra de casca foi colocada em uma estufa de convecção natural (modelo S250SD, fabricante Biopar, dimensões $60 \times 60 \times 70 \mathrm{~cm}$ ) sob temperatura fixa de $105 \pm 2^{\circ} \mathrm{C}$. Após 24 horas, essa amostra foi retirada, pesada e dividida entre os 21 frascos utilizados no experimento, de maneira que aproximadamente 2 gramas de casca fosse colocada em cada um. Esses foram fechados hermeticamente e deixados em outra estufa de marca Nova Ética na temperatura do experimento $\left(20,30,40,50\right.$ e $\left.60^{\circ} \mathrm{C}\right)$.

As amostras ficaram em condições termodinâmicas constantes, sendo feita a homogeneização das soluções em todas as ocasiões em que o material fosse pesado. Desta forma, considerou-se que as condições de equilíbrio foram alcançadas, quando a pesagem das amostras fornecessem três 
valores consecutivos idênticos. As pesagens foram realizadas em balança analítica, marca Marte, modelo AY220 com precisão de $0,0001 \mathrm{~g}$.

Com os dados da umidade de equilíbrio, $\mathrm{M}_{\mathrm{eq}}$, da umidade relativa do ar, UR e da temperatura do experimento, $\mathrm{T}_{\mathrm{s}}$, pôde-se ajustar os parâmetros de cada equação apresentada na Tabela 2 pelo método dos mínimos quadrados.

Tabela 2 - Equações para a umidade de equilíbrio.

\begin{tabular}{|c|c|c|}
\hline Nome & Equação & Referência \\
\hline Henderson & $M e q=\left[\frac{\ln (1-U R)}{-a T_{S}}\right]^{\frac{1}{b}}$ & Henderson (1952) \\
\hline Henderson - Thompson & $M e q=\left[\frac{\ln (1-U R)}{-a\left(T_{S}+c\right)}\right]^{\frac{1}{b}}$ & Thompson (1968) \\
\hline Chung - Pfost & $M e q=\frac{-1}{b} \ln \left[\frac{\left(T_{S}+c\right) \ln (U R)}{-a}\right]$ & Chung e Pfost (1967) \\
\hline Chen - Clayton & $M e q=\frac{-1}{c T_{S}^{d}} \ln \left[\frac{\ln (U R)}{-a T_{S}^{b}}\right]$ & Chen e Clayton (1971) \\
\hline Halsey modificada & $M e q=\left(\frac{-\exp \left(a T_{S}+c\right)}{\ln (U R)}\right)^{\frac{1}{b}}$ & Osborn et al.(1989) \\
\hline Oswin modificada & $M e q=\left(a+b T_{S}\right)\left(\frac{U R}{1-U R}\right)^{1 / c}$ & Chen e Morey (1989) \\
\hline Sabbah & $M e q=a \frac{U R^{b}}{T_{S}{ }^{c}}$ & Pfost et al (1976) \\
\hline Copace & $M e q=\exp \left(a-b T_{S}+c U R\right)$ & Corrêa et al (1995) \\
\hline Sigma-Copace & $M e q=\exp \left[a-b T_{S}+c \exp (U R)\right]$ & Corrêa et al (1995) \\
\hline
\end{tabular}

Para encontrar a equação que melhor representa o comportamento experimental da umidade de equilíbrio foi adotado um procedimento estatístico de discriminação de modelos rivais, que tem como base medidas de não linearidade que avaliam as propriedades estatísticas dos estimadores dos mínimos quadrados. As medidas de não linearidade utilizadas foram as de curvatura de Bates e Watts (1980) e de vício de Box (1971). As medidas de curvatura de Bates e Watts indicam o grau de não linearidade intrínseca de um modelo e o grau de não linearidade devido à parametrização utilizada, respectivamente. Os vícios de Box são utilizados para avaliar o vício dos parâmetros estimados por mínimos quadrados de modelos não lineares e, assim, indicar os parâmetros responsáveis pela não linearidade. 


\section{RESULTADOS E DISCUSSÃO}

Os resultados obtidos para o teor de umidade de equilíbrio da casca (base seca) encontram-se reunidos na Tabela 3, onde estão apresentadas as réplicas de cada situação operacional, bem como o respectivo desvio padrão amostral. Pode-se perceber que alguns valores de umidade de equilíbrio foram desprezados, visto que apresentavam grandes diferenças se comparados com os outros dados experimentais obtidos para uma mesma solução salina.

Analisando-se esses dados e o desvio padrão amostral médio $(0,1709)$, foi possível observar uma pequena variância das respostas medidas.

Tabela 3 - Teor de umidade de equilíbrio da casca, em base seca, e o desvio padrão amostral das réplicas para cada condição experimental.

\begin{tabular}{|c|c|c|c|c|c|}
\hline \multirow{4}{*}{$\begin{array}{c}\text { Solução } \\
\text { Salina }\end{array}$} & \multicolumn{5}{|c|}{ Temperatura $\left({ }^{\circ} \mathrm{C}\right)$} \\
\hline & 20 & 30 & 40 & 50 & 60 \\
\hline & \multicolumn{5}{|c|}{ Umidade de Equilíbrio } \\
\hline & \multicolumn{5}{|c|}{ (kg água/kg amostra seca) x 100} \\
\hline \multirow{4}{*}{$\mathrm{LiCl}$} & 2,08995 & 1,58213 & 2,15941 & 1,79177 & 2,47774 \\
\hline & 1,96929 & 1,96950 & 2,35373 & 1,76057 & 2,03202 \\
\hline & - & - & 1,93480 & 1,49086 & - \\
\hline & $\mathrm{s}=0,08531$ & $\mathrm{~s}=0,27390$ & $s=0,20965$ & $s=0,16546$ & $\mathrm{~s}=0,31517$ \\
\hline \multirow{4}{*}{$\mathrm{CH}_{3} \mathrm{CO}_{2} \mathrm{~K}$} & 3,79415 & 3,43206 & 3,55629 & 2,91987 & 3,17659 \\
\hline & 3,48370 & 3,29298 & 3,56118 & 3,02147 & 3,02065 \\
\hline & 3,43105 & 3,21781 & 3,59572 & 3,01259 & 2,89145 \\
\hline & $\mathrm{s}=0,19621$ & $\mathrm{~s}=0,10870$ & $\mathrm{~s}=0,02149$ & $\mathrm{~s}=0,05627$ & $s=0,14278$ \\
\hline \multirow{4}{*}{$\mathrm{MgCl}_{2}$} & 5,48952 & 4,05558 & 4,31342 & 3,81843 & 3,54680 \\
\hline & 5,68472 & 4,27899 & 4,05410 & 3,94977 & 3,78819 \\
\hline & - & 4,04821 & 4,19101 & 3,90577 & - \\
\hline & $\mathrm{s}=0,13802$ & $\mathrm{~s}=0,13117$ & $\mathrm{~s}=0,12972$ & $s=0,06684$ & $s=0,17069$ \\
\hline \multirow{4}{*}{$\mathrm{K}_{2} \mathrm{CO}_{3}$} & 9,49609 & 7,64048 & 5,58438 & 5,39643 & 4,71719 \\
\hline & - & 7,88118 & 5,58842 & 5,08508 & 5,14304 \\
\hline & - & & 5,93607 & 5,40971 & \\
\hline & - & $\mathrm{s}=0,17020$ & $s=0,20189$ & $s=0,18371$ & $\mathrm{~s}=0,30111$ \\
\hline \multirow{4}{*}{$\mathrm{NaNO}_{2}$} & 9,58710 & 8,15505 & 7,97423 & 7,44007 & 6,66699 \\
\hline & 9,36875 & 8,11668 & 8,45475 & 7,49557 & 6,73662 \\
\hline & 9,74987 & - & - & 7,31645 & - \\
\hline & $\mathrm{s}=0,19123$ & $\mathrm{~s}=0,02713$ & $\mathrm{~s}=0,33978$ & $\mathrm{~s}=0,09169$ & $s=0,04924$ \\
\hline \multirow{4}{*}{$\mathrm{NaCl}$} & 11,39784 & 11,18961 & 11,27713 & 10,83623 & 10,50882 \\
\hline & 11,79082 & 10,89512 & 11,31432 & 10,80338 & 10,29263 \\
\hline & - & - & - & - & - \\
\hline & $\mathrm{s}=0,27788$ & $\mathrm{~s}=0,20823$ & $\mathrm{~s}=0,02630$ & $\mathrm{~s}=0,02322$ & $\mathrm{~s}=0,15286$ \\
\hline
\end{tabular}




\begin{tabular}{c|c|c|c|c|c} 
& 14,56463 & 13,78510 & 12,10533 & 12,48808 & 12,05628 \\
& - & 13,53801 & 12,95483 & 12,37230 & 12,04396 \\
$\mathrm{KCl}$ & - & - & 11,11933 & - & - \\
& & & & & \\
& - & $\mathrm{s}=0,17472$ & $\mathrm{~s}=0,91859$ & $\mathrm{~s}=0,08187$ & $\mathrm{~s}=0,00871$ \\
\hline
\end{tabular}

Observa-se a partir da Tabela 3 que a umidade de equilíbrio $\left(\mathrm{M}_{\mathrm{eq}}\right)$ aumenta com a elevação da atividade da água (UR), demonstrando a higroscopicidade das amostras. A Tabela 4 apresenta os resultados

da estimação de parâmetros para as nove equações listadas na Tabela 2, bem como o coeficiente de determinação, as medidas de curvatura intrínseca (IN) e paramétrica (PE) e o vício de Box.

Tabela 4 - Resultado de mínimos quadrados e das medidas de curvatura e vício de Box para as equações de equilíbrio.

\begin{tabular}{|c|c|c|c|c|c|}
\hline Modelo & $\mathrm{R}^{2}$ & Curvatura & Parâmetro & $\begin{array}{c}\text { Valor } \\
\text { Estimado }\end{array}$ & $\begin{array}{c}\text { \% de Vício de } \\
\text { Box }\end{array}$ \\
\hline \multirow{2}{*}{ Henderson* } & \multirow[t]{2}{*}{0,8061} & $\mathrm{IN}=0,0738$ & $\mathrm{a}$ & 5,14147 & 17,8429 \\
\hline & & $\mathrm{PE}=4,4778$ & $\mathrm{~b}$ & 2,12233 & 0,8562 \\
\hline \multirow{3}{*}{$\begin{array}{l}\text { Henderson- } \\
\text { Thompson** }\end{array}$} & \multirow{3}{*}{0,9814} & \multirow{3}{*}{$\begin{array}{c}\mathrm{IN}=0,0484 \\
\mathrm{PE}=349,283\end{array}$} & $\mathrm{a}$ & 0,02811 & 3,4411 \\
\hline & & & b & 1,37612 & 0,0682 \\
\hline & & & $\mathrm{c}$ & 955,89249 & 179,4078 \\
\hline \multirow{3}{*}{$\begin{array}{l}\text { Chung- } \\
\text { Pfost** }\end{array}$} & \multirow{3}{*}{0,9764} & \multirow{3}{*}{$\begin{array}{c}\mathrm{IN}=0,2816 \\
\mathrm{PE}=849585,400\end{array}$} & $\mathrm{a}$ & 1752,03296 & 85,2367 \\
\hline & & & $\mathrm{b}$ & 21,74126 & 357738,8000 \\
\hline & & & $\mathrm{c}$ & 512,33389 & 92,0416 \\
\hline \multirow{4}{*}{$\begin{array}{c}\text { Chen- } \\
\text { Clayton } * * *\end{array}$} & \multirow{4}{*}{0,9771} & & $\mathrm{a}$ & 6,78410 & 12,6764 \\
\hline & & $\mathrm{IN}=0,1181$ & $\mathrm{~b}$ & $-0,20368$ & 0,6674 \\
\hline & & $\mathrm{PE}=14,5493$ & $\mathrm{c}$ & 37,77463 & 3,9832 \\
\hline & & & $\mathrm{d}$ & $-0,14673$ & 0,1785 \\
\hline \multirow{3}{*}{$\begin{array}{c}\text { Halsey } \\
\text { modificada** }\end{array}$} & \multirow{3}{*}{0,9891} & \multirow{3}{*}{$\begin{array}{c}\mathrm{IN}=0,02040 \\
\mathrm{PE}=0,0798\end{array}$} & $\mathrm{a}$ & $-0,00094$ & 0,0201 \\
\hline & & & $\mathrm{b}$ & 1,31921 & 0,0414 \\
\hline & & & $\mathrm{c}$ & $-4,01626$ & 0,0373 \\
\hline \multirow{3}{*}{ Copace** } & \multirow{3}{*}{0,9867} & \multirow{3}{*}{$\begin{array}{l}\mathrm{IN}=0,0241 \\
\mathrm{PE}=0,0461\end{array}$} & $\mathrm{a}$ & $-3,92304$ & 0,0171 \\
\hline & & & $\mathrm{b}$ & 0,00094 & $-0,1386$ \\
\hline & & & $\mathrm{c}$ & 2,40740 & 0,0274 \\
\hline \multirow{3}{*}{$\begin{array}{c}\text { Oswin } \\
\text { Modificada** }\end{array}$} & \multirow{3}{*}{0,9763} & \multirow{3}{*}{$\begin{array}{l}\mathrm{IN}=0,1648 \\
\mathrm{PE}=0,2639\end{array}$} & $\mathrm{a}$ & 0,06456 & $-0,1320$ \\
\hline & & & $\mathrm{b}$ & 0,000003 & 23,3888 \\
\hline & & & c & 2,01143 & 0,0492 \\
\hline \multirow{3}{*}{ Sabbah** } & \multirow{3}{*}{0,9736} & \multirow{3}{*}{$\begin{array}{l}\mathrm{IN}=0,0459 \\
\mathrm{PE}=2,2453\end{array}$} & $\mathrm{a}$ & 0,16927 & 0,9285 \\
\hline & & & $\mathrm{b}$ & 1,06547 & 0,0896 \\
\hline & & & $\mathrm{c}$ & 0,02826 & $-0,7250$ \\
\hline \multirow{3}{*}{$\begin{array}{l}\text { Sigma- } \\
\text { Copace** }\end{array}$} & \multirow{3}{*}{0,9886} & \multirow{3}{*}{$\begin{array}{l}\mathrm{IN}=0,0251 \\
\mathrm{PE}=0,0462\end{array}$} & $\mathrm{a}$ & $-5,26070$ & 0,0185 \\
\hline & & & $\mathrm{b}$ & 0,00039 & $-0,3830$ \\
\hline & & & $\mathrm{c}$ & 1,48788 & 0,0247 \\
\hline
\end{tabular}

Ao analisar a Tabela 4, pode-se notar que apenas para o modelo de Chung-Pfost, a medida de curvatura intrínseca (IN) foi significativa (valor maior que $1 / 2 \sqrt{F}$ ), mostrando assim que para este caso a não linearidade pode ser expressiva. Entretanto ao observar a medida paramétrica (PE), pôde-se verificar que as equações de Henderson, Henderson-Thompson, Chung-Pfost, ChenClayton, Oswin Modificada e Sabbah apresentam valores maiores que $1 / 2 \sqrt{F}$, indicando que pelo menos um dos parâmetros, 
de cada equação, apresenta comportamento não linear. Os maiores valores de vício de Box indicam qual parâmetro ou quais parâmetros provocam tal comportamento. As equações de Halsey Modificada, Copace e Sigma-Copace apresentaram vícios de Box não significativos (valores baixos) para todos os seus três parâmetros, bem como as medidas de curvatura de Bates e Watts não expressivos e bons coeficientes de determinação.

\section{CONCLUSÃO}

Através dos estudos realizados pode-se concluir que os modelos de Halsey Modificada, Copace e Sigma-Copace são os mais adequados para predizer a umidade de equilíbrio da casca da macadâmia. Isto pode ser comprovado pelos altos valores encontrados para $\mathrm{R}^{2}$, bem como os valores da curvatura intrínseca, paramétrica e vício de Box não significativos.

\section{NOMENCLATURA}

$\mathrm{M}_{\mathrm{eq}}$ : umidade de equilíbrio [ $\mathrm{kg}_{\text {água }} / \mathrm{kg}_{\text {amostra seca }}$ ] Ts: temperatura do experimento $\left[{ }^{\circ} \mathrm{C}\right]$ UR: umidade relativa $\quad\left[\mathrm{kg}_{\text {água }} / \mathrm{kg}_{\text {água saturação] }}\right.$

\section{REFERÊNCIAS}

Barrozo, M. A. S. (1995), Transferência de calor e massa entre o ar e semente de soja em leito deslizante e escoamentos cruzados, UFSCar, São Paulo-SP (tese de doutorado).

Bates, D.M.; Watts, D.G. (1980), Relative curvature measures of nonlinearity. Journal of the Royal Statistical Society Series B, vol. 42, p. 1-25.

Box, M. J. (1971), Bias in nonlinear estimation. Journal of the Royal Statistical Society Series B, vol. 33, p. 171-201.

Chen, C. C.; Morey, R. V. (1989), Comparison of four EMC/ ERH equations. Transactions of the ASAE, 32, 983-990.

Chen, C. S.; Clayton, J. T. (1971), The effect of temperature on sorption isotherms of biological materials. Transactions of the ASAE, vol 15, p. 927-929.
Chung, D.S.; Pfost, H.B. (1967), Adsorption and desorption of water vapors by cereal grains and their products Part II. Transactions of the ASAE, vol.10, p. 549551.

Corrêa, P. C.; Martins, D. S. R.; Melo, E. C. (1995), Umigrãos: programa para o cálculo do teor de umidade de equilíbrio para os principais produtos agrícolas. Viçosa: Centreinar - UFV, 10 p.

GLOBO RURAL. Cultivo da noz macadâmia cresce no Espírito Santo. Agronegócios. Rio de Janeiro, 19 abril 2011. Disponível em:

<http://g1.globo.com/economia/agronegoc ios/ noticia/2011/04/cultivo-da-nozmacadamia-cresce-no-espiritosanto.html>. Acesso em: 26 mar. 2012.

Henderson, S. M. (1952), A basic concept $\mathrm{f}$ equilibrium moisture content. Agricultural Engineering, vol. 33, p. 29-31.

Osborn, G. S.; White, G. M.; Sulaiman, A. H.; Welton, L. R. (1989), Predicting equilibrium moisture proportions of soybeans. Transactions of the ASAE, vol. 32, p. 2109-2113.

Pfost, H. B.; Maurer, S. G.; Chung, D. S.; Milliken, G. (1976), A. Summarising and reporting equilibrium moisture data for grains. ASAE Paper No. 76- 3520, ASAE, St. Joseph, Michigan, USA.

Pimentel, L. D. (2007), A cultura da macadâmia. Revista Brasileira de Fruticultura. v. 29. n. 3. Jaboticabal.

Poinern, G. E. J, Senanayake, G., Shah, N., Thi-le, X. N., Parkinson, G. M., Fawcett, D. (2011) Adsorption of the aurocyanide, complex on granular activated carbons derived from macadamia nut shells - A preliminary study. Minerals Engineering, v. 24, p. 1694-1702.

Thompson, T. L.; Peart, R. M.; Foster, G. H. (1968), Mathematical simulation of corn drying - a new model. Transactions of the ASAE, vol. 11, p. 582-586.

\section{AGRADECIMENTOS}

Os autores agradecem a CAPES, ao $\mathrm{CNPq}$ e à FAPES pelo apoio financeiro. 